\title{
Structure of the type IV secretion system in different strains of Anaplasma phagocytophilum
}

\author{
Basima Al-Khedery ${ }^{1}$, Anna M Lundgren ${ }^{1}$, Snorre Stuen², Erik G Granquist ${ }^{2}$, Ulrike G Munderloh³ ${ }^{3}$ Curtis M Nelson³, \\ A Rick Alleman ${ }^{4}$, Suman M Mahan ${ }^{5}$ and Anthony F Barbet ${ }^{1^{*}}$
}

\begin{abstract}
Background: Anaplasma phagocytophilum is an intracellular organism in the Order Rickettsiales that infects diverse animal species and is causing an emerging disease in humans, dogs and horses. Different strains have very different cell tropisms and virulence. For example, in the U.S., strains have been described that infect ruminants but not dogs or rodents. An intriguing question is how the strains of A. phagocytophilum differ and what different genome loci are involved in cell tropisms and/or virulence. Type IV secretion systems (T4SS) are responsible for translocation of substrates across the cell membrane by mechanisms that require contact with the recipient cell. They are especially important in organisms such as the Rickettsiales which require T4SS to aid colonization and survival within both mammalian and tick vector cells. We determined the structure of the T4SS in 7 strains from the U.S. and Europe and revised the sequence of the repetitive virB6 locus of the human $\mathrm{HZ}$ strain.

Results: Although in all strains the T4SS conforms to the previously described split loci for vir genes, there is great diversity within these loci among strains. This is particularly evident in the virB2 and virB6 which are postulated to encode the secretion channel and proteins exposed on the bacterial surface. VirB6-4 has an unusual highly repetitive structure and can have a molecular weight greater than 500,000. For many of the virs, phylogenetic trees position A. phagocytophilum strains infecting ruminants in the U.S. and Europe distant from strains infecting humans and dogs in the U.S.

Conclusions: Our study reveals evidence of gene duplication and considerable diversity of T4SS components in strains infecting different animals. The diversity in virB2 is in both the total number of copies, which varied from 8 to 15 in the herein characterized strains, and in the sequence of each copy. The diversity in virB6 is in the sequence of each of the 4 copies in the single locus and the presence of varying numbers of repetitive units in virB6-3 and virB6-4. These data suggest that the T4SS should be investigated further for a potential role in strain virulence of $A$. phagocytophilum.
\end{abstract}

Keywords: Anaplasma, phagocytophilum, Rickettsiales, T4SS, Comparative genomics

\section{Background}

Anaplasma phagocytophilum is a tick-borne pathogen in the Order Rickettsiales that is increasingly recognized as a cause of disease in humans and animals world-wide $[1,2]$. It causes the potentially fatal disease of human granulocytic anaplasmosis, which typically manifests as a flu-like illness accompanied by leukopenia, thrombocytopenia and anemia. It was initially recognized in the early 1990's when patients from Wisconsin and Minnesota

\footnotetext{
* Correspondence: barbet@ufl.edu

'Department of Infectious Diseases and Pathology, College of Veterinary

Medicine, University of Florida, Gainesville, FL, USA

Full list of author information is available at the end of the article
}

developed febrile illness following a tick bite [3]. Since that time the number of human cases has increased annually; between 2000 and 2007 the reported incidence in the U.S. increased from 1.4 to 3.0 cases/million persons/year [4]. The case fatality rate was $0.6 \%$ and the hospitalization rate was 36\%. In Massachusetts during the 2009 transmission season there were 33 confirmed cases with 14 (42\%) requiring hospitalization [5]. The human disease is also present in Europe and Asia [2]. A recent study of 83 A. phagocytophilum-infected patients in China reported a mortality rate in this cohort of $26.5 \%$ [6]. In the U.S., there has been a parallel increase in cases of the disease [7] and seroprevalence [8] in dogs in the eastern and

\section{Biomed Central}


upper Midwestern states. The tick vectors in the U.S. are Ixodes scapularis and Ixodes pacificus and wild rodents are the main reservoirs of human infections. A. phagocytophilum also infects numerous other mammalian species including ruminants, horses, cats, and bears and the symptoms are extremely variable, with some mammalian species exhibiting acute disease and others only persistent asymptomatic infections $[9,10]$. For example, A. phagocytophilum strains isolated from deer in the U.S. can have a slightly different $16 \mathrm{~S}$ rRNA sequence and be uninfective to mice and it is thought, humans [11-13]. In Europe, this agent has been known to cause disease of ruminants for $>100$ years, yet there have been few human infections [14]. The genome sequence is available for a single strain of $A$. phagocytophilum derived from an infected human in the U.S. and it is apparent that, although this strain lacks Type II, III, $\mathrm{V}$ and VI secretion systems, a Type IV secretion system (T4SS) is present [15]. As in other members of the Rickettsiales, the T4SS of A. phagocytophilum is organized differently from most gram-negative bacteria with the component vir genes distributed between three major genome locations [16].

The T4SS typically encodes a membrane-spanning multiprotein complex that forms a transmembrane channel through which solutes can pass into host cells. It can mediate transfer of DNA and proteins into eukaryotic host cells, interfere with host signaling, and is essential for the survival of intracellular bacteria [17]. In A. phagocytophilum, which preferentially colonize neutrophilic white blood cells, it is thought that the T4SS secretes virulence factors that are responsible for subverting innate immunity and inhibiting host cell apoptosis [16]. Interestingly, there appears to be differential transcription of the T4SS in ticks and in the mammalian host with virB6 and virB9 upregulated during infection of human neutrophils and different virB2 paralogs expressed in mammalian and tick cells [18]. There is evidence that VirB2, VirB6 and VirB9 are exposed on the outer membrane surface in the Rickettsiales [18-20], which has stimulated interest in their potential use as vaccine candidates. This possibility has been investigated more extensively in the related organism Anaplasma marginale [21-25]. In A. marginale, unlike many other surface-exposed proteins, the T4SS proteins are conserved between strains [26]. Also, cattle immunized with outer membranes and protected against challenge infection respond with IgG and $\mathrm{T}$ cells to Vir proteins, notably VirB2, VirB9 and VirB10. To date, only two T4SS substrates have been identified and partially characterized in A. phagocytophilum: the ankyrin repeat domaincontaining protein, AnkA, and the Anaplasma translocated substrate 1, Ats-1. AnkA translocates to the host nucleus and interacts with DNA [27,28], while Ats- 1 is imported into the mitochondria where it is proposed to interfere with the induction of apoptosis [29].

In this study, we compared the structure and diversity of the T4SS in different strains of A. phagocytophilum infecting humans, dogs, rodents and ruminants. Most diversity was found in the proteins thought to be surfaceexposed, which may be associated with the different virulence and cell invasion properties of this species.

\section{Results and discussion}

The vir loci were sequenced in eight strains of $A$. phagocytophilum; seven of these were strains for which previous structural information was not available and included organisms originally isolated from U.S. dogs (ApDog1, $A p \operatorname{Dog} 2)$, a rodent $(A p \mathrm{JM})$, a horse (ApMRK), the ruminant $A p$ variant 1 strain ( $A p \operatorname{Var} 1)$ and two strains from Norwegian sheep (ApNorV1, ApNorV2). The human HZ strain was also resequenced, as optical mapping had suggested a possible error in the previously sequenced virB6-4 locus. The data indicated considerable diversity in the individual vir loci between strains that will be discussed below. In all strains, however, as noted previously [20,30], the vir loci were distributed mainly in three gene clusters comprising: virB8-1, virB91, virB10, virB11 and virD4; virB2/s and virB4-2; and $\operatorname{virB3}$, virB4-1, and the four virB6 paralogs (Figure 1). These three loci may each be transcribed polycistronically [31], although it is clear that T4SS structure in the Rickettsiales is unique and more complex than initially thought. The number of virB2 paralogs was different between strains with the human HZ strain having the least (8 total paralogs) and the ruminant strains having the most (up to15 total paralogs). The description of the T4SS components presented here follows the functional classification described by Alvarez-Martinez and Christie [20].

\section{Inner membrane channel/scaffold subunits: VirB3, VirB6, VirB8 and VirB10}

The most conserved of these subunits are VirB3, VirB8 and VirB10, with few differences between strains. VirB3 has been linked in Agrobacterium tumefaciens with pilus assembly and substrate translocation [32,33]. It is absolutely conserved between strains with no amino acid changes and conforms to the typical VirB3 structure. Two alpha-helical domains for insertion into the cytoplasmic membrane are strongly predicted by TMpred. VirB8, proposed to function as a nucleation factor during the assembly of T4SS $[34,35]$, is also well conserved, particularly VirB8-1 in the polycistronic transcription locus (one amino acid change between all strains). VirB10, proposed to function as a scaffold across the entire cell envelope [36], is also generally wellconserved with the exception of one ruminant strain, 


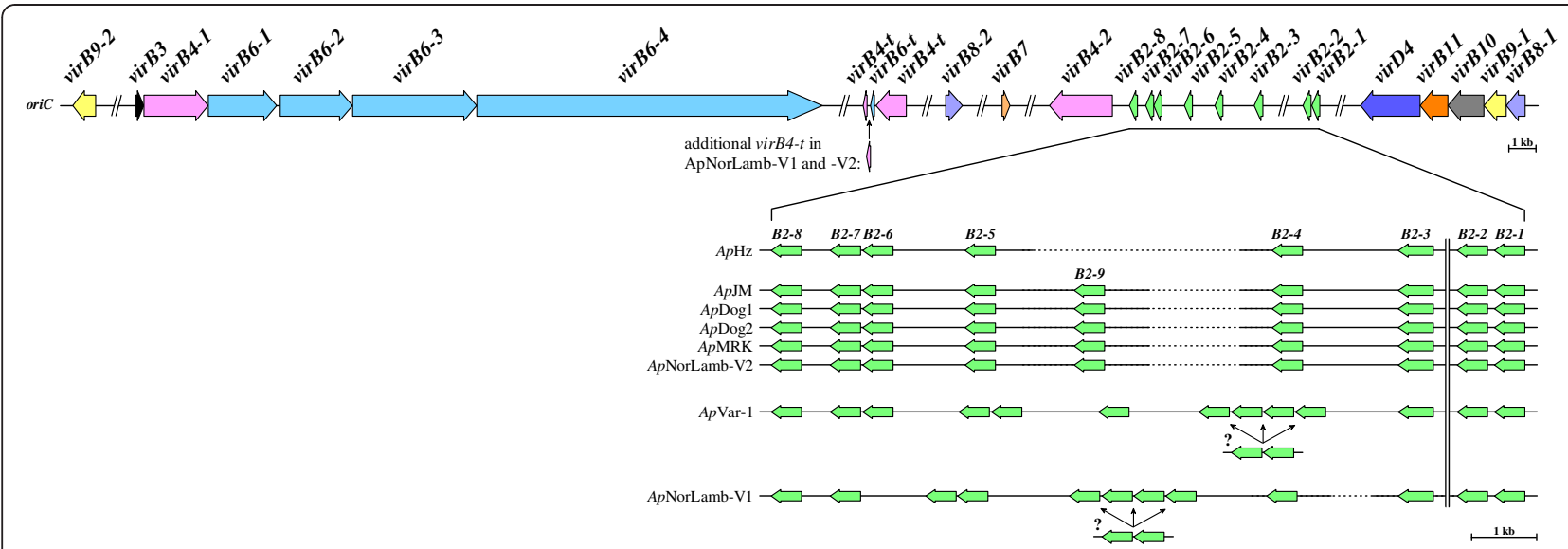

Figure 1 Distribution and content of vir gene clusters in eight diverse $A$. phagocytophilum strains. Top panel. Schematic representation of all vir loci (colored arrows) showing the three conserved gene cluster islands (see text). VirB-7, virB8-2 and virB9-2 are not part of vir gene clusters, but their location relative to surrounding genes is also highly conserved among strains. A small cluster comprising truncated ( $\mathrm{t}$ ) virB6 and virB4 gene fragments is present in all strains, but the Norwegian lamb strains have one additional virB4-t. Bottom panel. Magnification of the virB2 gene cluster. Numbering of paralogs $1-8$ is based on the original ApHZ annotated genome (GenBank CP000235). Artificial gaps (stippled lines) were introduced to allow alignment of the more spatially conserved paralogs B2-1, 2-2 and 2-3 at one end, and B2-7 and 2-8 at the other end of the cluster. With the exception of virB2-9, lacking in $A p H Z$, the number and arrangement (but not necessarily sequence) of virB2 genes is highly conserved in all but the US ruminant ApVar-1 and ApNorLamb-V1, which have several additional virB2 genes. In both strains a sub-cluster of 6 distinct genes was present. Due to the repetitive nature of sequences in this region, combined with the relatively short length of 454 reads ( $\leq 550 \mathrm{bp}$ ), their placement could not be confidently ascertained (highlighted by arrows and '?'). Maps are drawn to scale. Double lines designate interruption in sequences. Genes belonging to the same grouping have the same color. oric; origin of replication.

ApNorLamb-V1, which has 31 amino acid substitutions with respect to $A p H Z$ (data not shown). However, all $A$. phagocytophilum VirB10's, including ApNorLamb-V1, have two strongly predicted transmembrane domains, which supports their function as membrane scaffolding subunits in these organisms.

Of these inner membrane channel subunits, the data on VirB6 are the most interesting. All VirB6 subunits that have been described possess a highly hydrophobic membrane domain including five or more predicted transmembrane domains [20]. Some VirB6 proteins also have an extended C-terminal hydrophilic domain that has been proposed to protrude through the T4SS into the target cell, or may be proteolytically released from the N-terminal domain and then translocated into the target cell. Evidence has been obtained for surface

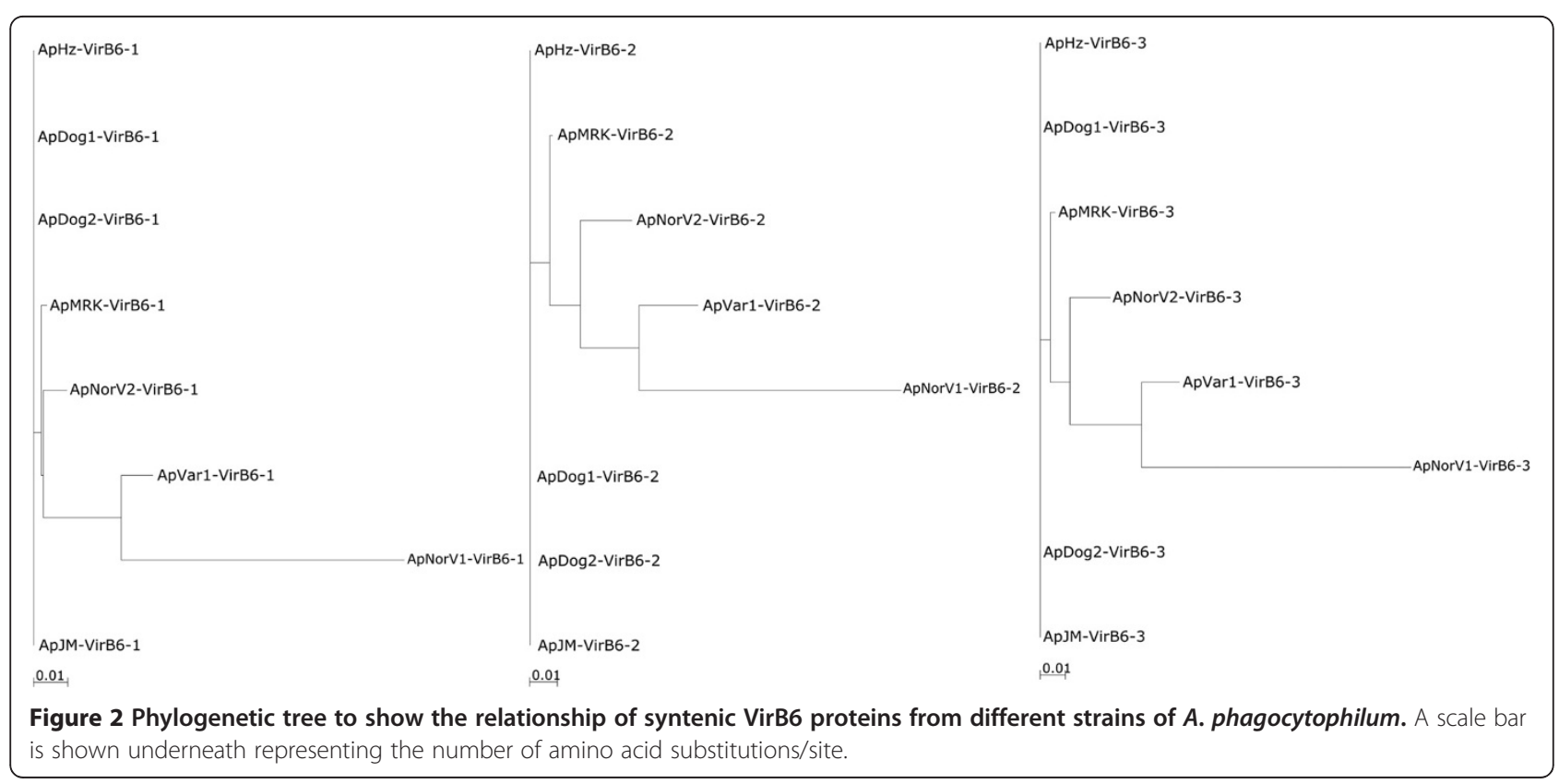


exposure of extended VirB6 in some Rickettsiales [37]. Of all the membrane channel subunits, the most sequence diversity between $A$. phagocytophilum strains was in the four VirB6 paralogs (Figure 1). Although there were no amino acid changes in the VirB6-1, VirB62 and VirB6-3 paralogs between human, dog and rodent strains, the ruminant and horse strains had numerous substitutions throughout each molecule, agreeing with the closer evolutionary relationship between strains infecting humans and dogs in the U.S. (Figure 2). Furthermore, major differences in repeat number and sequence were found in the $\mathrm{C}$-terminal repeat region of VirB6-3 (yellow boxes in Figure 3A and Additional file 1: Figure S1) in ruminant and horse strains, with the horse strain showing the least variability from $A p \mathrm{HZ}$.

The only amino acid differences detected between the human, dog and rodent strains were in the VirB6-4 subunit. VirB6-4 in these strains contains four repeat regions (R1-R4 in Figure 3A) and variability in repeat number, order and sequence were found mainly in R3 and R4 (Additional file 2: Figure S2). Within R1 (Figure 3A), the only difference detected was in ApDog2 which had 4 and 1 partial of 231 bp repeat units (data not shown), compared to 3 and 1 partial repeats in the $A p D o g 1, A p \mathrm{JM}$ and $A p \mathrm{HZ}$ virB6-4 R1. Optical mapping of the Dog1 genome and comparison with $A p \mathrm{HZ}$ suggested that the sequence obtained previously for the human HZ strain virB6-4 was incorrect (Figure 3B). This was confirmed by PCR and sequencing, and mapped specifically to the $3^{\prime}$-most $\mathrm{R} 4$ region (Figure $3 \mathrm{C}$ ). Because of its size and unusual composition it was only possible to resolve this sequence using the long readlength Pacific Biosciences technology (see Methods). The corrected virB6-4 R4 of $A p \mathrm{HZ}$, totaling $6.89 \mathrm{~kb}$, differed from the original by $5.88 \mathrm{~kb}$ of additional sequence composed exclusively of $84 \mathrm{bp}$ [type 1 , a and b (T1a, T1b); light/dark blue boxes, respectively, in Figure 3A] and 162 bp [type 2, a and b (T2a, T2b); light/dark orange boxes, respectively, in Figure 3A] repeat units, giving a complex repeat structure containing 53 and 1

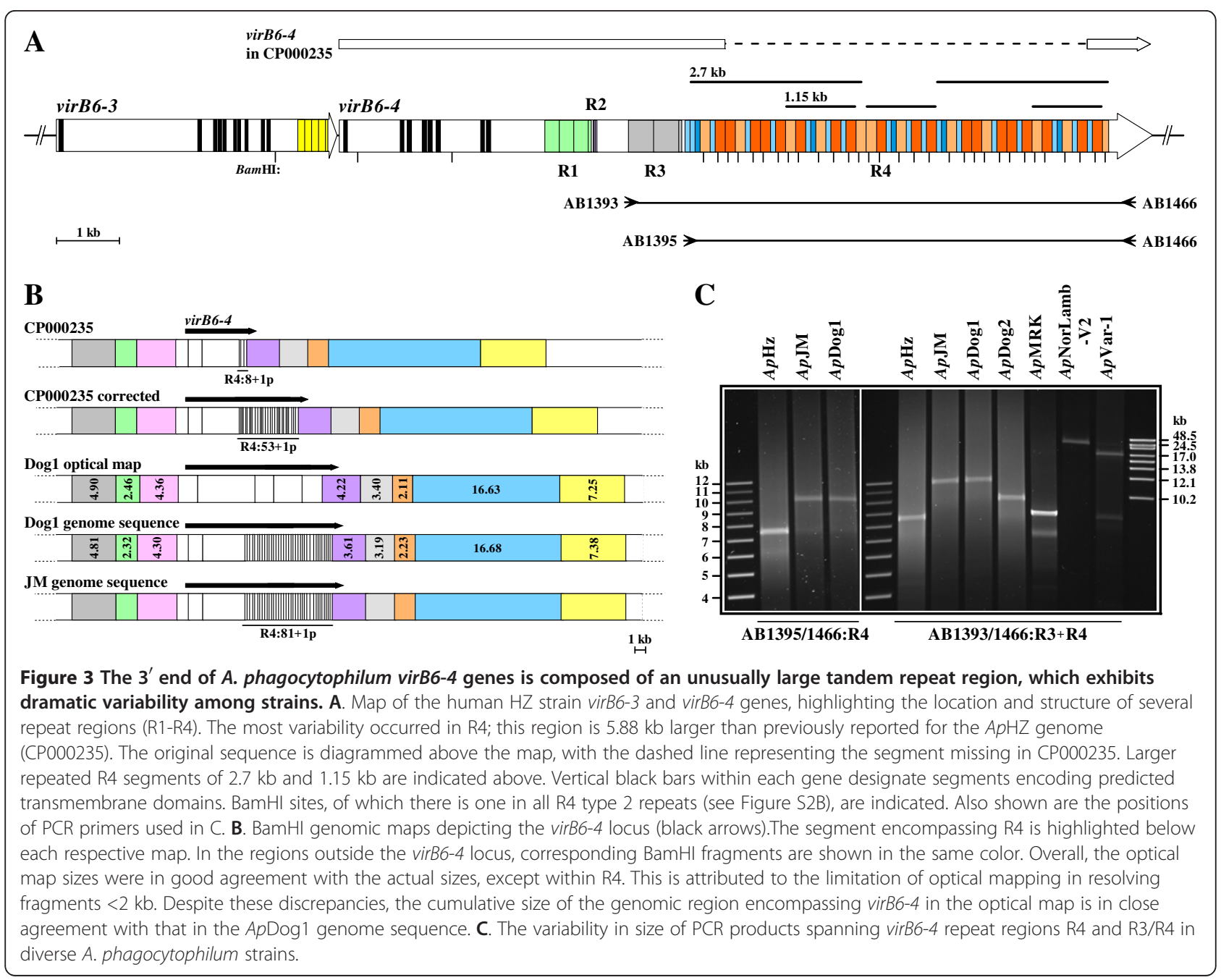


partial repeat units compared to 8 and 1 partial in the original sequence. Further, the $5^{\prime}$ - and $3^{\prime}$-most $2.7 \mathrm{~kb}$ of this complex structure are identical in sequence, and the $3^{\prime}$-most $1.15 \mathrm{~kb}$ of each of these segments is repeated again in the center of R4 (Figure 3A and Additional file 2: Figure S2). Although the possibility exists that the $A p \mathrm{HZ}$ population from which we isolated gDNA differs within the virB6-4 R3/R4 repeat regions from the population used to generate CP000235, the fact that all strains investigated herein presented expansive R3/R4 regions (Figure 3C) would contradict that. Instead, it is more plausible that the existence of $2.7 \mathrm{~kb}$ of identical repeats at the ends of the $A p \mathrm{HZ}$ R4 may have lead to the excision of most of its sequence during construction/propagation of those libraries. Interestingly, virB6-4 R3 and R4 were identical both in size and sequence in the Dog1 and rodent strains despite differing markedly from the $\mathrm{HZ}$ and Dog2 strain regions (Additional file 2: Figure S2A). Within R3, these strains had 2 additional 405 bp repeats compared to $\mathrm{ApHZ}$ and one more compared to the Dog2 strain. However, differences between strains were most dramatic within $\mathrm{R} 4$. Not only was this region in $A p \operatorname{Dog} 1 / A p \mathrm{JM} 2.87 \mathrm{~kb}$ larger than in $A p \mathrm{HZ}$ bringing the total number of repeats to 81 and 1 partial, but intriguingly, the repeat pattern was completely unrelated to that in the $\mathrm{HZ}$ strain. Also, the Dog1 and rodent strain R4 lacked T1b repeat units, while having a third type 2 repeat variant, namely T2c, which differed from T2b by 1 SNP and a 12 bp deletion (Additional file 2: Figure S2). Partial analysis of the ApDog2 454 reads spanning $\mathrm{R} 4$ (estimated at $\sim 8 \mathrm{~kb}$ by PCR; Figure $3 \mathrm{C}$ ) showed that the order of the $5^{\prime}$ - and $3^{\prime}$-most three repeat units differed from either the $\mathrm{HZ}$ or Dog1/rodent strain R4 repeat patterns (Additional file 2: Figure S2A). Notably, our preliminary analyses of the horse and ruminant 454 reads suggest the absence of distinct $\mathrm{R} 3$ and $\mathrm{R} 4$ regions in virB6-4 in these strains. Rather, the few repeat units identified to date appear to be a combination of R3 and R4 repeats (data not shown). It is also unclear if the $\sim 17 \mathrm{~kb}$ and $\sim 25 \mathrm{~kb}$ PCR products generated with primers AB1393/1466 in ApVar-1 and ApNorLamb-V2, respectively (Figure $3 \mathrm{C}$ ), are composed mainly of repeats, or alternatively if a fifth virB-6 gene paralog exists in these strains. Taken together, the data presented here clearly demonstrate the extreme variability of the T4SS VirB6-4 subunit among A. phagocytophilum strains. Although the differences between the more closely related human, dog and rodent US strains were mainly within repeat-laden regions, the fact that an extensive, distinct repeat pattern was maintained in two strains would speak against the possibility that the variability may be attributed solely to the highly recombinogenic nature of such structures. Worth noting, Camp Ripley, where the infected jumping mouse was captured (2001) is only $\sim 20$ miles away from the city of Baxter, MN, where Dog1 resides. Although there are no records of where this dog may have actually acquired the infection, it presented with severe clinical disease in 2007.

The unusual structure and likely antigenicity of the C-terminal region of the A. phagocytophilum VirB6-4's is apparent in hydrophobicity plots (Figure 4). What specific properties these distinct repeat patterns may confer onto each strain awaits functional analysis of these proteins in A. phagocytophilum. The corrected VirB6-4 translated protein had a predicted molecular weight of 470,695 Da containing 4,322 amino acid residues compared to molecular weights of 90,742, 103,204 and $158,321 \mathrm{Da}$ for the HZ strain VirB6-1, VirB6-2 and VirB6-3, respectively. Interestingly, the predicted acidity of the VirB6's also increased from VirB6-1 to VirB6-4 (pI's of 8.4, 6.8, 5.1 and 4.0 for the $A p H Z$ VirB6-1, VirB62, VirB6-3 and VirB6-4, respectively). The ApDog1/ApJM VirB6-4 polypeptides had a predicted molecular weight of 603,529 Da containing 5,550 amino acids, and a pI of 3.96. Despite these dissimilarities, at least eight transmembrane segments were predicted for all VirB6 paralogs.

\section{Periplasmic/outer membrane channel subunits: VirB2, VirB7 and VirB9}

Several other T4SS subunits contribute to the secretion channel across the periplasm and outer membrane. VirB7 subunits are typically small lipoproteins that may stabilize VirB9 [38,39]. In A. phagocytophilum strains a putative VirB7 is absolutely conserved between strains and may be lipid modified through an N-terminal cysteine on the mature molecule. VirB9 is hydrophilic and also localizes to the periplasm and outer membrane. In A. tumefaciens the C-terminal region of VirB9 is part of the outer membrane protein channel and is surface accessible [40]. There is also evidence for surface exposure of VirB9 in Ehrlichia chaffeensis and A. phagocytophilum $[18,19,41]$. VirB9-1, which is encoded on the polycistronic virB8-1-virD4 transcript [31], has a strongly predicted signal peptide and two transmembrane helices. Of all the potentially exposed components of the T4SS, VirB9 of $A$. phagocytophilum appears to be the least diverse among strains. There are some amino acid substitutions in ruminant and horse strains (2-6 total compared to $A p \mathrm{HZ})$ but in the other strains VirB9's are unchanged (data not shown).

Unlike VirB9's, VirB2's are the most diverse of all T4SS subunits in A. phagocytophilum, in terms of both copy number and sequence. VirB2 proteins are typically constituents of pili and of the secretion channel and their diversity in Anaplasma suggests the possibility of exposed, antigenically variable structures. In A. marginale, VirB2 is expressed together with the major outer 

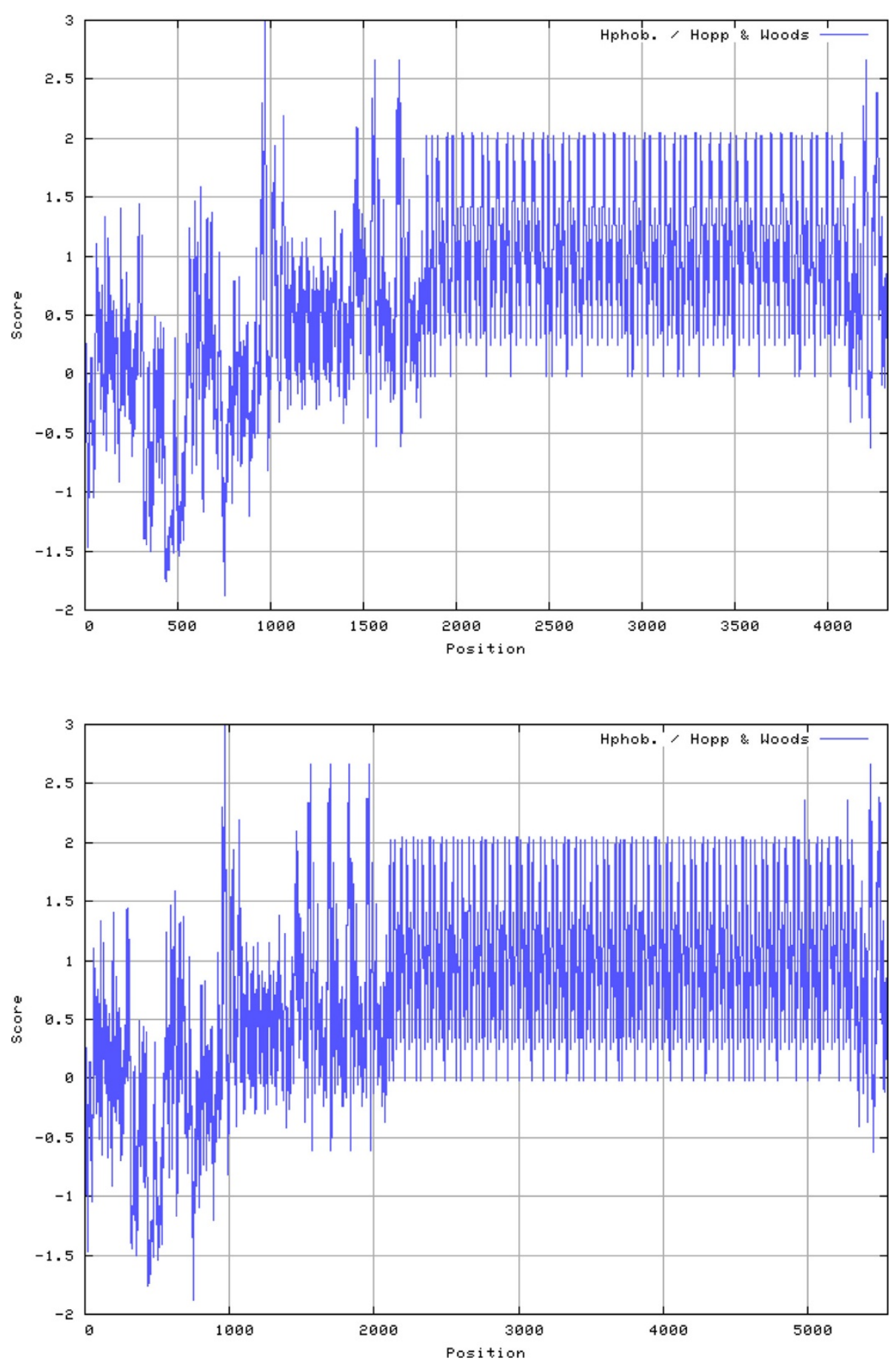

Figure 4 Hydrophobicity plots of VirB6-4 proteins from A. phagocytophilum HZ (top) or Dog 1 (bottom) strains.

membrane protein MSP3 on a sequence-variable polycistronic transcript $[25,42]$. The mechanism of expression in A. phagocytophilum is not known. VirB2's of other genera are typically small hydrophobic proteins with a long signal peptide sequence and two hydrophobic alpha helices for integration into the cytoplasmic membrane. This also appears to be the case for $A$. phagocytophilum. The VirB2 paralogs in the different strains are predicted to have two hydrophobic alphahelices of lengths $22+/-3$ and $20+/-0.2$ amino acids and signal peptides of length $27+/-2$ amino acids. This is true despite their sequence diversity (Figure 5). As with many other T4SS components, the ruminant and horse strains are more distant taxonomically in VirB2 


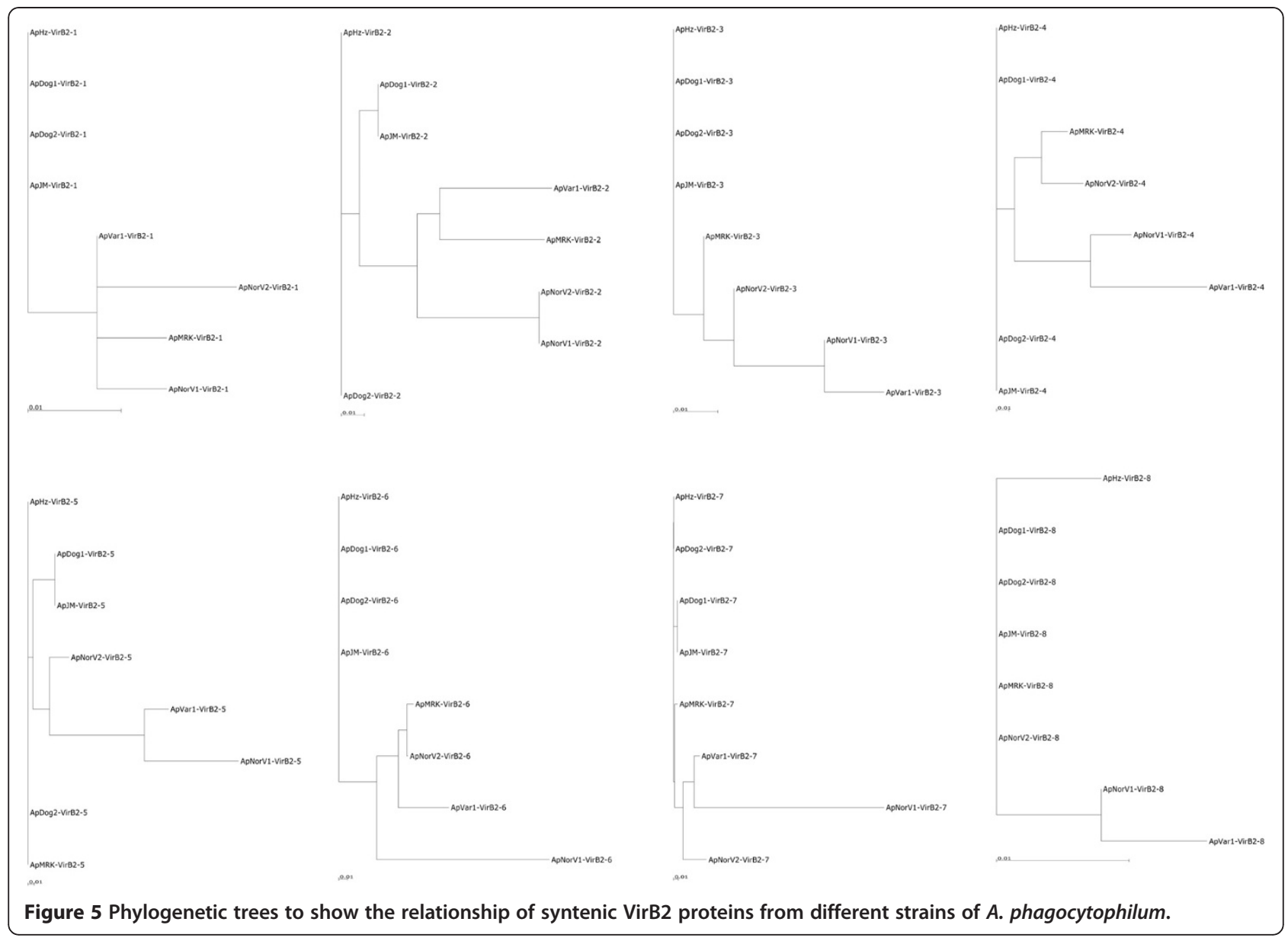

sequence compared to VirB2's of human and dog strains. Alignment of all VirB2 paralogs and orthologs shows that sequence diversity is primarily localized to two hypervariable regions either preceding an $\mathrm{N}$ terminal cysteine or close to the C-terminus (Figure 6). This is similar to the hypervariable regions found among VirB2 paralogs of $A$. marginale [25].

\section{Energetic subunits: VirB4 and VirB11}

ATPases are typically used in T4SS to energize substrate transfer and have been found in every T4SS described. In gram-negative bacteria these are typically integral membrane proteins encoded by genes residing upstream of virB2 (encoding pilin). This is true for all strains of $A$. phagocytophilum and it has been suggested that this arrangement of multiple virB2 paralogs and virB4-2 may allow assembly of an antigenically variable surface organelle [20]. The energetic subunit itself, VirB4-2, is however, well conserved between strains. The most distant taxonomic relationship was found between human and ruminant strains (29 total amino acid substitutions in $A p$ NorLamb-V1 compared to $A p \mathrm{HZ}$, Figure 7). The other energetic subunit, VirB11, was also well-conserved between strains ( 6 amino acid substitutions between $A p$ NorLamb-V1 and $A p \mathrm{HZ}$; data not shown).

\section{Type 4 coupling protein: VirD4}

Type 4 coupling proteins such as VirD4 are ATPases that function in substrate recognition and translocation using the T4SS. They are associated with most effector translocator systems. They typically possess a minimum of two N-terminal transmembrane domains. Often most heterogeneity exists in these $\mathrm{N}$-terminal regions [20]. The A. phagocytophilum VirD4's conform somewhat to this stereotype with three strongly predicted N-terminal transmembrane segments. As with the other ATPases of the A. phagocytophilum T4SS, there is little variation in VirD4, a total of 17 amino acid substitutions of which 4 are $\mathrm{N}$-terminal but more (12) are C-terminal. Again, the evolutionary relationships among VirD4 sequences position the ruminant and horse strains more distantly to the U.S. dog, human and rodent strains (Figure 8).

\section{Conclusions}

A. phagocytophilum represents a recent reclassification of intracellular organisms infecting different animal species 


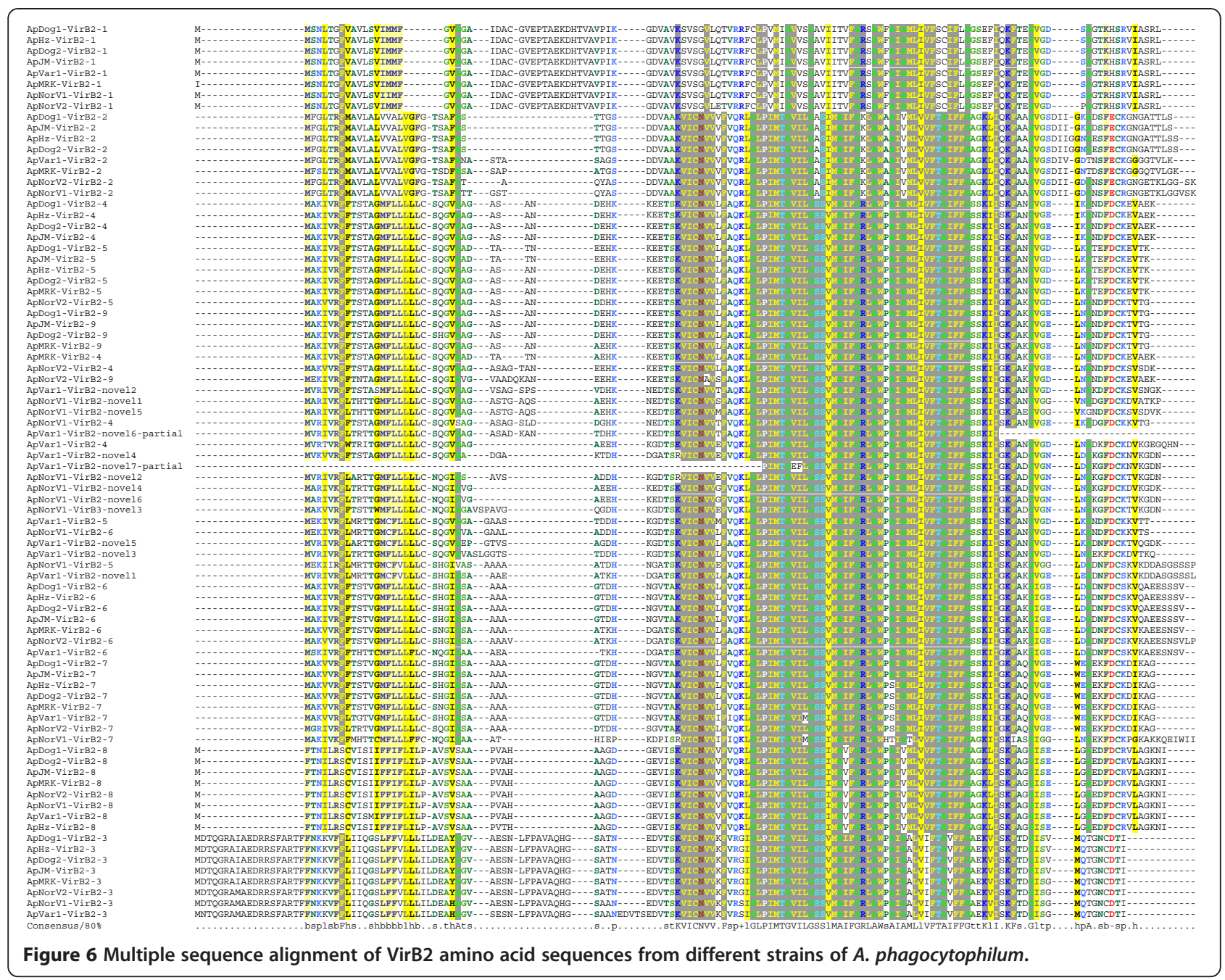

and humans and causing diverse disease symptomatology [43]. These bacteria were previously known as Ehrlichia phagocytophila, Ehrlichia equi, and the agent of human granulocytic ehrlichiosis. Despite the differences within this species, the overall genome structure and synteny of the T4SS is maintained. However, gene structural analysis reveals evidence of gene duplication and considerable diversity of T4SS components in strains infecting different animals. Taxonomic trees suggest a close evolutionary relationship of $A$. phagocytophilum strains infecting U.S. humans, mice and dogs and a more distant relationship with ruminant and horse strains. This relationship is not unique to the T4SS but is also supported by similar taxonomic trees of other A. phagocytophilum proteins of conserved metabolic function (Figure 9). Within the T4SS multicomponent membrane complex, the energetic and internal scaffolding protein components are the most conserved. In contrast, components that form the proposed exposed structures of the T4SS, such as VirB2 and VirB6, are more variable. T4SS are important virulence determinants of bacteria, therefore these differences may result in the different infectivity and virulence profiles observed with different strains. It will be of interest to determine the molecular architecture of VirB6 paralogs in different strains, including interactions with other T4SS components and effectors. Of the known surface exposed components of the T4SS, VirB9 is the most conserved. This protein has been proposed as a vaccine component against $A$. marginale and may also be suitable against A. phagocytophilum.

\section{Methods}

A. phagocytophilum strains, cell culture, and experimental infection

The A. phagocytophilum U.S. strains HZ (human-origin, NY), MRK (horse-origin, CA), JM (rodent-origin, MN) and Dog1 (dog-origin, MN) were propagated in HL-60 cells in RPMI-1640 medium (Thermo Fisher Scientific, Inc., Waltham, MA) supplemented with final 10\% heatinactivated fetal bovine serum (Thermo Scientific) and 


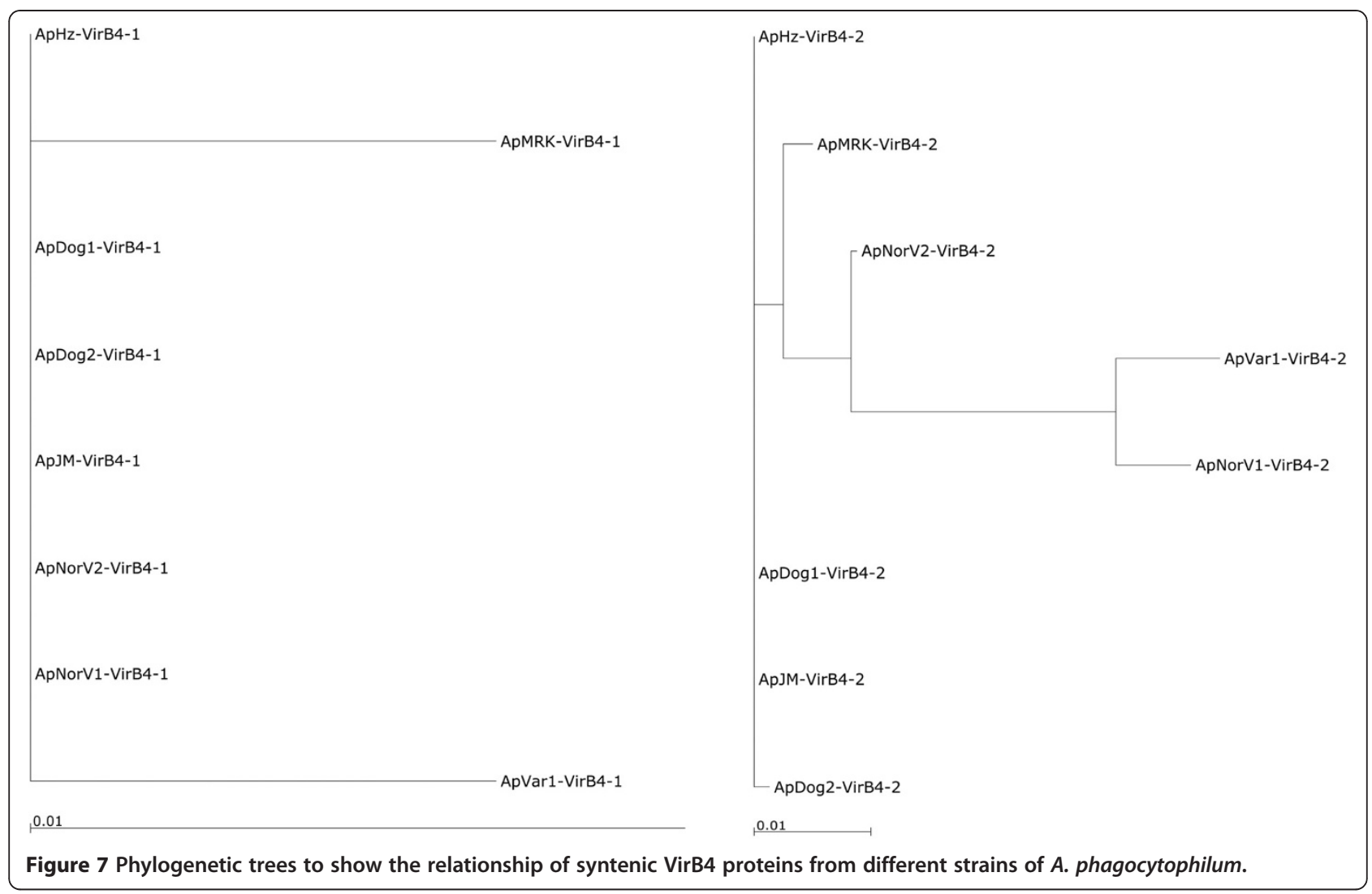

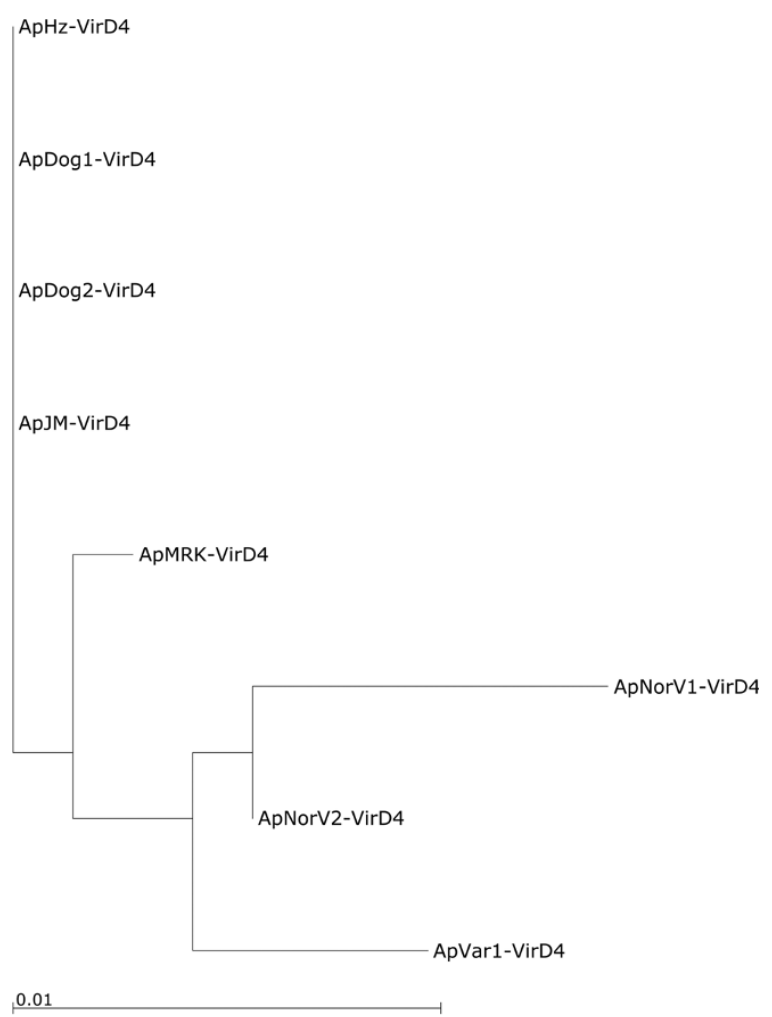

Figure 8 Phylogenetic tree to show the relationship of syntenic VirD4 proteins from different strains of $A$. phagocytophilum.
4 mM L-glutamine (Lonza, Rockland, ME), and in the absence of antibiotics. ApHZ and ApMRK have been described previously $[15,44]$. The $A p$ JM strain (CR011258 ) originated from a meadow jumping mouse (Zapus hudsonius) trapped at Camp Ripley, MN [45]. The $A p \operatorname{Dog} 1$ strain originated from the blood of a dog from Baxter, MN naturally infected with A. phagocytophilum, as evidenced by the detection of distinctive morulae in a diagnostic blood sample, and sequencing of the Expression Site-linked $m s p 2 / p 44$ gene. Briefly, whole blood was collected from the animal with EDTA as an anticoagulant. The buffy coat layer was collected after low-speed centrifugation of the whole-blood, washed in $1 \mathrm{x}$ phosphate buffered saline (PBS, Hyclone, cat. no. SH30256.01), then added to a culture of uninfected HL-60 cells. The culture was left undisturbed for 3 days, after which morulae began to appear. The $A p D o g 2$ strain also originated from a $\mathrm{MN}$ dog and was passaged to and maintained in the Ixodes scapularis ISE6 tick cell line as described [46]. The Ap variant 1 CRT35 strain (tick-origin, MN), maintained in ISE6 cells, has been described [47]. For DNA isolation, cultures were maintained until 90-100\% of cells were infected with mature morulae. Cells were pelleted by centrifugation at $2500 \mathrm{x} \mathrm{g}$ for $20 \mathrm{~min}$ at $4^{\circ} \mathrm{C}$. Pellets were gently resuspended in $1.5 \mathrm{ml}$ cold PBS, transferred to screw-cap microfuge tubes, and centrifuged at 


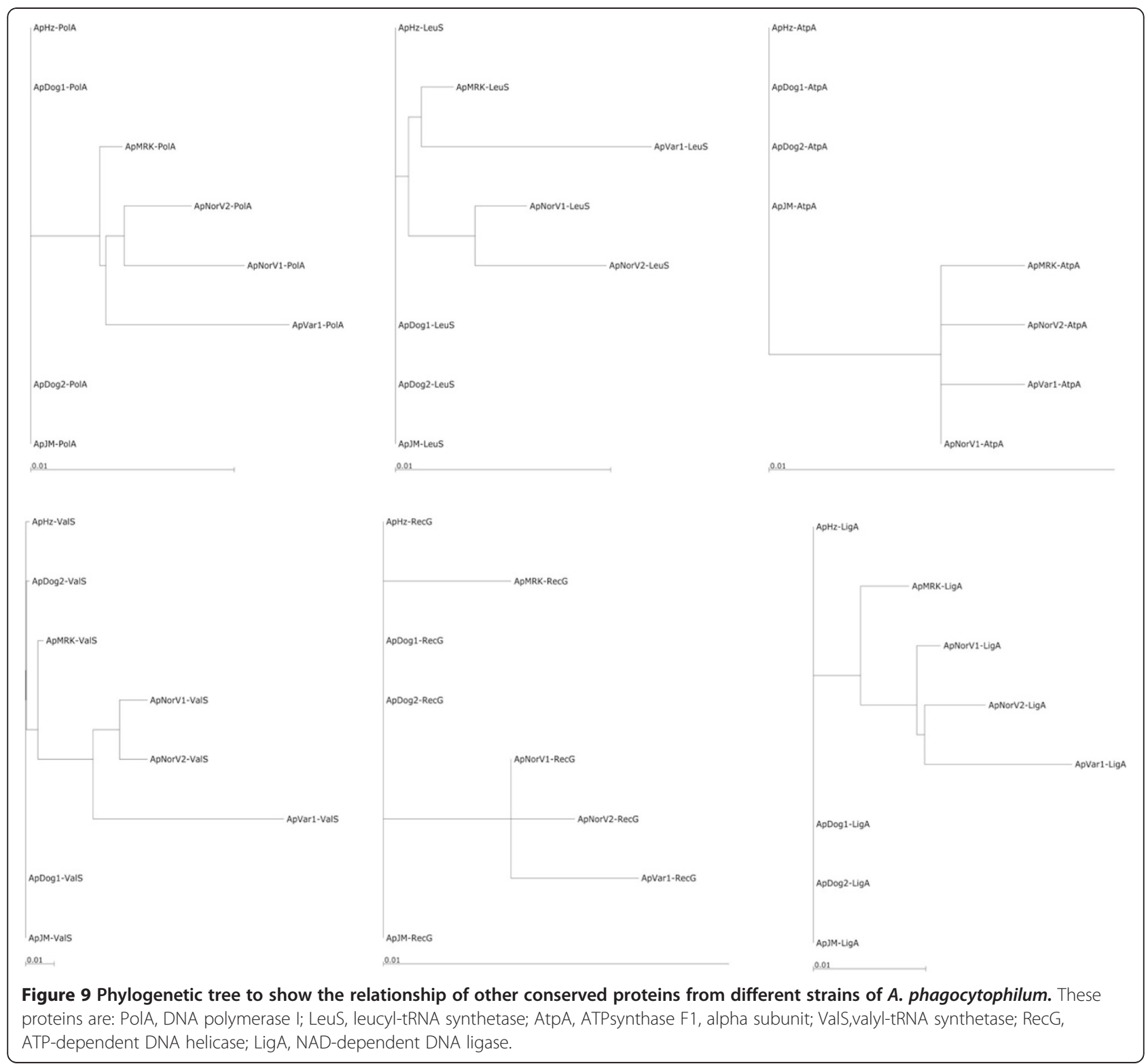

$1500 \mathrm{x} \mathrm{g}$ for $20 \mathrm{~min}$ at $4^{\circ} \mathrm{C}$. Supernatants were removed and the cell pellets stored at $-80^{\circ} \mathrm{C}$ until further use.

Two naturally occurring Norwegian lamb A. phagocytophilum strains differing in the $16 S$ rRNA gene and degree of virulence were used to experimentally infect lambs raised in an indoor environment with barriers against tick entry and tick infestation. Lamb 00186 was infected with the more virulent variant 1 (identical to GenBank M73220) and lamb 0054 with variant 2 (identical to GenBank AF336220) [48], to be referred to as $A p$ NorLamb-V1 and -V2 from here on. Infections were monitored by microscopy and blood was harvested at maximum parasitemia. To purify buffy coats containing the infected neutrophils, approximately 2.51 of $\mathrm{Na}$ citrated blood was collected from each animal. The blood was transferred to 11 centrifuge bottles and centrifuged at 2,500-3,000 $\mathrm{x} \mathrm{g}$ in a swing-out bucket rotor for $30 \mathrm{~min}$ at $4^{\circ} \mathrm{C}$. After removing most of the plasma layer, the buffy coat layer was collected with minimal contamination of red blood cells. The cells were diluted 1:3 with PBS, mixed gently and centrifuged at 1,500x g for $20 \mathrm{~min}$ at $4^{\circ} \mathrm{C}$. Following three PBS washes, supernatants were removed and the cell pellets stored at $-80^{\circ} \mathrm{C}$. The experimental study in sheep was approved by the Norwegian Animal Research Authority.

\section{Purification of host cell-free $A$. phagocytophilum and genomic DNA (gDNA) isolation}

For the HZ, JM, Dog1, MRK and NorLamb-V1 and -V2 strains, intact, host cell-free organisms with minimal 
host cell gDNA/RNA contamination were purified from frozen PBS pellets of infected cells prepared as above. Samples and reagents were maintained on ice throughout the entire procedure, and all centrifugations performed at $4^{\circ} \mathrm{C}$. Following a quick thaw, host cells were disrupted by vigorous vortexing for $5 \mathrm{~min}$. An equal volume of PBS was added and vortexing continued for 3 min. Cellular debris was removed by centrifugation at $200 \mathrm{x} g$ for $15 \mathrm{~min}$. After removing most of the supernatants to fresh tubes, these were passed several times through a $31 \mathrm{G}$ needle and saved on ice. Pellets were resuspended well in final $500 \mu \mathrm{l}$ PBS then passed serially through 22 G, 25 G, 28 G and, when possible, 31 G needles attached to a $1 \mathrm{ml}$ syringe. 3-5 volumes $\mathrm{PBS}$ were added and mixed by vortexing. Debris was removed by centrifugation at $200 \mathrm{x}$ g for $10 \mathrm{~min}$. Supernatants were pooled to those from the previous centrifugation step. RNaseA was added to a final $250-300 \mu \mathrm{g} / \mathrm{ml}$ and the samples incubated $45-60 \mathrm{~min}$ at $37^{\circ} \mathrm{C}$. Samples were centrifuged at $21,000 \times \mathrm{g}$ for $30 \mathrm{~min}$ and the supernatants removed completely. Pellets were resuspended in 50-100 $\mu \mathrm{l}$ PBS each and transferred to fresh tubes. To ensure homogeneity of the suspension, initially a drawnout $10 \mu \mathrm{l}$ pipette tip was used to disrupt the pellet by swirling followed by up/down pipetting and gentle vortexing, before switching to a larger tip. The sample was further homogenized by several passes through a 28$31 \mathrm{G}$ needle. PBS was added to final 500-700 $\mu \mathrm{l}$ and DNaseI to final $250 \mu \mathrm{g} / \mathrm{ml}$. Following 45-60 min incubation at $37^{\circ} \mathrm{C}$ the samples were centrifuged at $21,000 \times \mathrm{g}$ for $30 \mathrm{~min}$. Pellets were homogenized as above and the DNaseI treatment repeated. EDTA ( $\mathrm{pH} \mathrm{8.0)} \mathrm{was} \mathrm{added}$ to final $25 \mathrm{mM}$ and the samples centrifuged as above. Tubes were washed twice with PBS without disturbing the pellets and residual PBS was removed after $3 \mathrm{~min}$ centrifugation at $21,000 \mathrm{x}$ g. Pellets were homogenized as above in 600-800 $\mu \mathrm{l}$ RPMI culture medium (containing $10 \%$ fetal bovine serum) added incrementally and transferred to a $50 \mathrm{ml}$ tube. Culture medium was added to a final volume of $6 \mathrm{ml}$ before passage through a prewet, $2 \mu \mathrm{m}$ pore-size, $25 \mathrm{~mm}$, GMF-150 glass microfiber syringe filter (Puradisc 25GD; Whatman Inc., Florham Park, NJ). The filter was washed $3-4 \mathrm{x}$ with culture medium. Washes were pooled to the filtrate and centrifuged at 22,000 x g for $30 \mathrm{~min}$. The pellets, comprised of free, non-viable organisms and host cell mitochondria, were resuspended in PBS, transferred to microfuge tubes and re-pelleted at $21,000 \mathrm{x}$ g for $30 \mathrm{~min}$. Supernatants were removed completely and the pellets were processed immediately or stored at $-20^{\circ} \mathrm{C}$. For every $10^{8}$ host cells used at $90-100 \%$ infectivity, enough organisms were recovered to yield on average 1-1.5 $\mu \mathrm{g}$ high-quality DNA using either the Gentra Puregene Yeast/Bact. kit (Qiagen Inc., Valencia, CA) or the QIAGEN Blood \&
Cell Culture DNA mini kit following the manufacturer's protocols.

For the Dog2 and Ap variant 1 strains, organisms were cultured and isolated from ISE6 tick cells as described [49]. Host cell-free bacteria were prepared from two cultures in $25 \mathrm{~cm}^{2}$ flasks, collected by centrifugation for $10 \mathrm{~min}$ at $11,000 \mathrm{xg}$ at $4^{\circ} \mathrm{C}$, and lysed in Gentra Puregene lysis buffer (Qiagen) at $80^{\circ} \mathrm{C}$ for $5 \mathrm{~min}$. Since these DNA samples also contained a considerable amount of small $(<500 \mathrm{bp})$ DNA species naturally associated with the ISE6 host cell line, the $A$. phagocytophilum gDNA was further purified by electroelution from agarose gels, followed by phenol/chloroform extraction and $\mathrm{EtOH}$ precipitation using conventional protocols.

\section{Preparation of host cell-free A. phagocytophilum agarose plugs for optical mapping}

ApDog1 was initially selected for complete genome sequencing to compare with the published $\mathrm{HZ}$ strain. When a draft genome was assembled for $A p D o g 1$ it was largely syntenic with $\mathrm{HZ}$ except for the virB6 locus, indicating a possible error in the sequence of one or both of the strains. Accordingly, the $A p \operatorname{Dog} 1$ draft genome sequence was verified by Optical Mapping. In preparation for Optical Mapping (performed by OpGen Inc., Gaithersburg, MD), host cell-free organisms were embedded in $0.5 \%$ low-melting point agarose plugs and subsequently lysed, allowing access to the intact, $\sim 1.48 \mathrm{Mb}$ circular A. phagocytophilum chromosome. A procedure recommended by OpGen was followed. All solutions were made fresh using OpGen suggested reagents. Intact $A p D o g 1$ organisms were purified as above, except that the pellet of free organisms obtained following centrifugation of the filtrate was resupended and washed in cell suspension buffer [200 $\mathrm{mM} \mathrm{NaCl}$, $100 \mathrm{mM}$ EDTA-Na $\mathrm{Na}_{2}$ (pH 8.0), $10 \mathrm{mM}$ Tris ( $\mathrm{pH}$ 7.2)]. Plugs were made immediately on completion of the isolation procedure. Briefly, following the final centrifugation of the purified organisms, the pellet was resuspended in cell suspension buffer using 40-50 $\mu \mathrm{l}$ for every $10^{8}$ host cells used at $>95 \%$ infectivity. The sample was passed $2 \mathrm{x}$ through a $31 \mathrm{G}$ needle $(3 / 10 \mathrm{ml}$ capacity Insulin Syringe with fused $8 \mathrm{~mm}$ long needle, BD \#328438; Becton, Dickinson \& Co., Franklin Lakes, NJ) to ensure homogeneity of the thick suspension, and an equal volume of $1 \%$ low melting point SeaPlaque GTG agarose [(Lonza \#50111) dissolved in DEPC-treated water (Invitrogen \#750023; Carlsbad, CA) and held at $55^{\circ} \mathrm{C}$ ] was immediately added. Following mixing, $100 \mu \mathrm{l}$ aliquots were dispensed into plug molds (Bio-Rad \#170-3713; Hercules, CA) and allowed to set for $1 \mathrm{hr}$ at $4^{\circ} \mathrm{C}$ prior to transfer into a $50 \mathrm{ml}$ tube containing $5-10 \mathrm{ml}, 50^{\circ} \mathrm{C}$ NDSK solution [filter sterile NDS solution (1\% N-lauroylsarcosine (Sigma \#L5000; St. Louis, MO) in $0.5 \mathrm{M}$ EDTA-Na 
(pH 9.5), supplemented with final $2 \mathrm{mg} / \mathrm{ml}$ proteinase K (Pierce \#17916; Rockford, IL) immediately prior to use]. The tube was incubated upright at $50^{\circ} \mathrm{C}$ with mild shaking $(40 \mathrm{rpm})$ for $8-24 \mathrm{hrs}$, until the plugs turned clear and colorless. Plugs were gently washed $3 \mathrm{x}$ in $5 \mathrm{ml} \mathrm{0.5} \mathrm{M}$ EDTA- $\mathrm{Na}_{2}$ (pH 9.5), then transferred to a fresh tube and stored in EDTA at $4^{\circ} \mathrm{C}$. Optical Mapping data generated from the BamHI-digested ApDog1 chromosome was analyzed using the OpGen MapSolver software.

\section{Genome sequencing and bioinformatics}

Isolated DNA was provided to the Interdisciplinary Center for Biotechnology Research (ICBR) core facilities, University of Florida for library construction and pyrosequencing on the Roche/454 Genome Sequencer according to standard manufacturer protocols. Regular read libraries were generated for all strains. Additionally, $3 \mathrm{~kb}$ paired end libraries were made for $A p \mathrm{HZ}, A p D o g 1$ and ApMRK. Genome coverage range was $31.3 \mathrm{x}$ to $72.1 \mathrm{x}$. For each strain, the SFF format flow files, returned by ICBR for bioinformatics analysis, were first combined and converted to .fasta and .qual files (or the two combined in .fastq format) using Roche/454 Genome Sequencer FLX System software. Genome drafts were assembled using the CLC Genomics Workbench software suite (version 4.0-4.9) by mapping reads initially against the fully annotated, Sanger sequenced $A p \mathrm{HZ}$ genome (GenBank CP000235), then against the completed $A p D o g 1$ genome. Default parameters were used: length fraction, 0.5; similarity, 0.8; and for paired end reads, minimum distance, 1500/maximum distance, 4500. To obtain the vir loci, the resulting consensus sequence and underlying aligned reads were inspected for conflicts and mismatched paired ends suggesting the presence of insertions and/or deletions not mirrored in the consensus. These were manually corrected. Gaps were also manually closed where possible. Briefly, overlapping reads covering at least $2 \mathrm{~kb}$ of sequence on both sides of a gap and extending into it were individually extracted from the alignment. A new consensus for each side was obtained by assembling the reads against each other, and 250 N's were added to its ends. These were individually used as the reference sequence against which all the 454 reads were re-mapped to pull out novel reads extending into the unknown region. The process was repeated multiple times, allowing for the incremental filling of the gap. PCR, followed by sequencing was performed when sequences extrapolated in this fashion spanned complex tandem repeat regions such as repeat regions 1 and 3 ( $\mathrm{R} 1$ and $\mathrm{R} 3$ in Figure 3A) of the virB6-4 gene, or when gap closure could not be completed due to such structures, as was the case with the extremely long virB6-4 R4 (Figure 3A) region.
Amino acid sequences were aligned with MAFFT [50] and displayed with CHROMA [51]. Taxonomic relationships used a neighbor-joining tree and the ITT substitution model [52] and were displayed using Archaeopteryx (http://www.phylosoft.org/archaeopteryx). Hydrophobicity analyses were conducted using the method of Hopp and Woods [53,54] at web.expasy.org and transmembrane segments were predicted with TMpred at http://www.ch. embnet.org/software/TMPRED_form.html.

PCR amplification of virB6-4 gene repeat regions, cloning, and Pacific Biosciences sequencing

Due to difficulties in amplifying tandem repeat-containing DNA, all PCR reactions spanning the virB6-4 gene repeat regions were performed in the presence of 1.5-1.7 M Betaine (Sigma). The $8.36 \mathrm{~kb}$ PCR product spanning R3 and $\mathrm{R} 4$ in the $A p \mathrm{HZ}$ strain (Figure 3A, 3C, and Additional file 2: Figure $\mathrm{S} 2 \mathrm{~A}$ ) was amplified using the iProof High-Fidelity DNA Polymerase system with GC buffer (Bio-Rad). Reactions totaled $50 \mu \mathrm{l}$ with $5 \mathrm{ng}$ purified $A$. phagocytophilum gDNA, $1.0 \mathrm{U}$ polymerase, $1.5 \mathrm{mM} \mathrm{MgCl}_{2}$, $200 \mu \mathrm{M}$ each dNTP, and $250 \mathrm{nM}$ each primer (AB1393: 5'-CGGGATCTAAGACAGATGATGATTC-3', forward; AB1466: 5'-CTCATCCTGATGCGTCTCCTTAG-3', reverse; Figure $3 \mathrm{~A}) .35$ cycles of $30 \mathrm{sec}$ denaturing at $98^{\circ} \mathrm{C}$, $20 \mathrm{sec}$ annealing at $67^{\circ} \mathrm{C}$, and $5 \mathrm{~min}$ extension at $72^{\circ} \mathrm{C}$ were performed. PCR products spanning $\mathrm{R} 4$ in $A p \mathrm{JM}$ and ApDog1 (both $\sim 10.3 \mathrm{~kb}$; Figure $3 \mathrm{C}$ ) were derived using Takara's PrimeSTAR GXL DNA Polymerase system (Clontech Laboratories, Mountain View, CA). Reactions contained $5 \mathrm{ng}$ gDNA, $1.25 \mathrm{U}$ polymerase, $1.0 \mathrm{mM} \mathrm{MgCl}_{2}$, $200 \mu \mathrm{M}$ each dNTP, and $200 \mathrm{nM}$ each primer (AB1395: $5^{\prime}-$ CACCAGAGGATGCAGCATTAG-3', forward; AB1466, reverse; Figure $3 \mathrm{~A}$ ) in total $50 \mu \mathrm{l}$. Following the manufacturer's recommendations, 2-step PCR was performed with 30 cycles of $10 \mathrm{sec}$ denaturing at $98^{\circ} \mathrm{C}$ and $10 \mathrm{~min}$ annealing/extension at $68^{\circ} \mathrm{C}$. PCR products were analyzed on $0.5 \%$ agarose gels alongside the $1 \mathrm{~kb}$ Plus (Invitrogen) and the GeneRulerHighRange (Fermentas, Inc., Glen Burnie, MD) DNA ladders. In order to TA-clone the amplicons, Aoverhangs were added to the ends using 0.5-1.0 units AmpliTaq DNA polymerase (Applied Biosystems, Foster City, CA) in a $10-15 \mathrm{~min}$ reaction at $72^{\circ} \mathrm{C}$. Products purified from agarose gels (before or after A-overhang addition) were cloned into the pCR-XL-TOPO vector (Invitrogen) and transformed into E. coli Stbl2 (Invitrogen), which is more permissive to repeat-laden foreign DNA. Recombinants containing the correct size insert were end sequenced to verify their identity.

In preparation for sequencing with the long-read length Pacific Biosciences (PacBio) next-generation sequencing RS instrument, constructs were linearized with restriction enzymes which cut the vector only, but on opposite sides of the insert within the Multiple Cloning 
Site. For $A p \mathrm{HZ}$, equimolar amounts of the TA clone were cut with either HindIII or EcoRV. Following pooling and EtOH precipitation, the linearized DNA mix was submitted to ICBR/UF for SMRTbell library construction and sequencing. Libraries were constructed using a commercial strobe library preparation kit (\#001-326-530; Pacific Biosciences, Menlo Park, CA) following standard manufacturer protocols. To further increase the likelihood of full coverage, the strobesequencing run was performed using two different conditions: I) 45 min light period (continuous collection time); and II) (5 min light period, $10 \mathrm{~min}$ dark period), followed by (45 min light period, 10 min dark period). The $A p \mathrm{JM}$ and $A p \operatorname{Dog} 1$ constructs were double-digested with HindIII/XbaI to excise the $\sim 10.3 \mathrm{~kb}$ inserts. Following separation on $0.5 \%$ agarose gels, the inserts were recovered from agarose slices by electroelution and further purified and concentrated by passage over QIAquick spin columns following the PCR Purification kit protocol (Qiagen). SMRTbell libraries were made as above then sequenced using a single $75 \mathrm{~min}$ movie time run.

Due to the repetitive nature of the cloned gene fragments, combined with the relatively high error-rate of the PacBio system, all attempts to assemble the reads de novo failed to yield a sequence of the expected size. Therefore, for each construct, reads $>3 \mathrm{~kb}$ were selected from the multi-fasta files using the Galaxy suite [55], and imported into the CLC Genomics Workbench for assembly and further analysis. These were assembled at low stringency initially against a consensus sequence representing an entire (vector and insert sequence) linear construct to which sufficient N's were added based on the estimated gap-size. Starting with reads initiating outside the repeat region, the longest of the assembled reads were visually inspected for the presence of virB6-4 R4 repeat signature-sequences (Additional file 2: Figure S2) and their sequence manually corrected where necessary. The extended sequences were used to replace N's in the consensus and the process repeated several times until sufficient reads with $>2 \mathrm{~kb}$ sequence overlap were recovered spanning the entire insert region. For verification, the completed sequence for each strain was used as the reference to re-map all the respective $>3 \mathrm{~kb}$ PacBio reads and the Roche/454 reads at higher stringency.

\section{GenBank Accession Numbers: for each isolate, the vir genes are listed in order}

The sequences of vir loci are complete for strains $A p \operatorname{Dog} 1$ and $A p J M$. The sequence of the repetitive virB6-4 locus was incomplete $(A p D o g 2)$ or not determined for the other strains except $A p \mathrm{~Hz}$. We provide a revised sequence of virB6-4 for the previously sequenced [15] ApHZ strain.
ApDog1:JX415845 - JX415868

B2-1 B2-2, B2-3, B2-4, B2-5, B2-6, B2-7, B2-8, B2-9, B3, B4-1, B4-t1, B4-2, B6-1, B6-2, B6-3, B6-4, B8-1, B82, B9-1, B9-2, B10, B11, D4

ApJM:JX415869 - JX415892

B2-1, B2-2, B2-3, B2-4, B2-5, B2-6, B2-7, B2-8, B2-9, B3, B4-1, B4-t1, B4-2, B6-1, B6-2, B6-3, B6-4, B8-1, B82, B9-1, B9-2, B10, B11, D4

ApDog2:JX415893 - JX415915 (virB6-4 submitted separately as gapped)

B2-1, B2-2, B2-3, B2-4, B2-5, B2-6, B2-7, B2-8, B2-9, B3, B4-1, B4-t1, B4-2, B6-1, B6-2, B6-3, B8-1, B8-2, B91, B9-2, B10, B11, D4

ApNorLambV2:JX415916 - JX415938

B2-1, B2-2, B2-3, B2-4, B2-5, B2-6, B2-7, B2-8, B2-9,

B3, B4-1, B4-t1, B4-2, B6-1, B6-2, B6-3, B8-1, B8-2, B91, B9-2, B10, B11, D4

ApNorLambV1:JX415939 - JX415966

B2-1, B2-2, B2-3, B2-4, B2-5, B2-6, B2-7, B2-8, B2novel1, B2- novel2, B2-novel3, B2-novel4, B2-novel5, B2-novel6, B3, B4-1, B4- t1, B4-2, B6-1, B6-2, B6-3, B81, B8-2, B9-1, B9-2, B10, B11, D4

ApHZvirB6-4:JX415967

Ap Var1:JX415968 - JX415996

B2-1, B2-2, B2-3, B2-4, B2-5, B2-6, B2-7, B2-8, B2novel1, B2- novel2, B2-novel3, B2-novel4, B2-novel5, B2-novel6, B2-novel7, B3, B4-1, B4-t1, B4-2, B6-1, B6-2, B6-3, B8-1, B8-2, B9-1, B9-2, B10, B11, D4

ApMRK:JX415997 - JX416019

B2-1, B2-2, B2-3, B2-4, B2-5, B2-6, B2-7, B2-8, B2-9, B3, B4-1, B4-t1, B4-2, B6-1, B6-2, B6-3, B8-1, B8-2, B91, B9-2, B10, B11, D4

ApDog2virB6-4Gapped:JX416020.

\section{Additional files}

Additional file 1: Figure S1. Multiple sequence alignment of VirB6-3 amino acid sequences from different strains of A. phagocytophilum. Arrows indicate the locations of C-terminal 41-mer repeats.

Additional file 2: Figure S2. Structure of the virB6-4 repeat regions R3 and R4 in four US A.phagocytophilum strains.A. Comparative maps of AB1393/AB1466 PCR products detailing the repeat unit content of R3 and R4 in the human, rodent and dog strains. ApJM and ApDog1 have identical virB6-4 genes and are, therefore, represented by one map. Moderate variability in the number and sequence of the R3 405 bp repeat units (light blue arrows) is apparent. The small bar at the end of R3 corresponds to the $3^{\prime}$-most partial repeat unit present in all strains. 
The colored arrows within R4 represent the five repeat types T1a (yellow), T1b (green), T2a, (red), T2b (dark blue) and T2c (grey). The repeat pattern in $\mathrm{ApHZ}$ shows no relationship to that of $A p J \mathrm{M} / \mathrm{ApD} D$ og 1, which is also $2.87 \mathrm{~kb}$ larger, totaling $9.76 \mathrm{~kb}$. This region was not fully characterized in ApDog2 as indicated by a broken line, but the repeat pattern of the $5^{\prime}$ and $3^{\prime}$-most repeats is clearly different from that of the other strains. The small bar downstream of the second repeat unit represents a partially characterized type 2 repeat unit. Lines above and below the $\mathrm{ApHZ}$ and ApJM/ApDog1 maps delineate segments of sequence identity within the respective R4 regions. Their sizes are specified. B. Alignment of the nucleic acid sequence of all virB6-4 R4 repeat unit types identified to date. Type 1 repeats are shown in black, type 2 in blue. Differences between sub-types are highlighted. A single BamHI site present in all type 2 repeats is underlined. With the exception of only a few nucleotides at each end, type 1 and type 2 repeat units do not share any sequences. C. Alignment of the amino acid sequences of the repeat units shown in B. The single nucleotide differences between sub-types do not lead to changes in amino acid sequence.

\section{Competing interests}

The authors declare that they have no competing interests.

\section{Authors' contributions}

BAK and AFB conceived the study, performed bioinformatics analyses and drafted the manuscript. BAK grew infected HL-60 cell cultures, purified organisms, isolated gDNA, designed and supervised PCR and submitted sequences to GenBank. AML performed PCR analyses and cloning and supervised data transfer between units. SS and EGG isolated the European sheep strains, infected and monitored sheep, and prepared organisms at maximal parasitemia. UGM and CMN isolated and cultured in vitro the $\mathrm{JM}$ MRK, Dog2 and Ap variant 1 strains, and prepared Dog2 and Ap variant 1 strain gDNA. ARA and SMM established the Dog1 strain. All authors read and approved the final manuscript.

\section{Acknowledgements}

The research described here received support from grants RO1 GM081714 and GM081714-03S1 and from Pfizer Animal Health. We thank Dr. Roberta Veluci-Marlow, Susan Benda and Adam Webster for help with culturing cells infected with A. phagocytophilum, and Dr. Savita Shanker for highthroughput DNA sequencing.

\section{Author details}

'Department of Infectious Diseases and Pathology, College of Veterinary Medicine, University of Florida, Gainesville, FL, USA. ${ }^{2}$ Department of Production Animal Sciences, Section of Small Ruminant Research, Norwegian School of Veterinary Science, Sandnes, Norway. ${ }^{3}$ Department of Entomology, University of Minnesota, St Paul, MN, USA. ${ }^{4}$ Physiological Sciences, College of Veterinary Medicine, University of Florida, Gainesville, FL, USA. ${ }^{5}$ Pfizer Animal Health, Kalamazoo, MI, USA.

Received: 10 July 2012 Accepted: 20 November 2012

Published: 29 November 2012

\section{References}

1. Dumler JS, Choi KS, Garcia-Garcia JC, Barat NS, Scorpio DG, Garyu JW, et al: Human granulocytic anaplasmosis and Anaplasma phagocytophilum. Emerg Infect Dis 2005, 11:1828-1834.

2. Jin H, Wei F, Liu Q, Qian J: Epidemiology and control of human granulocytic anaplasmosis: a systematic review. Vector Borne Zoonotic Dis 2012, 12:269-274.

3. Bakken JS, Dumler JS: Clinical diagnosis and treatment of human granulocytotropic anaplasmosis. Ann N Y Acad Sci 2006, 1078:236-247.

4. Dahlgren FS, Mandel EJ, Krebs JW, Massung RF, McQuiston JH: Increasing incidence of Ehrlichia chaffeensis and Anaplasma phagocytophilum in the United States, 2000-2007. Am J Trop Med Hyg 2011, 85:124-131.

5. Weil AA, Baron EL, Brown CM, Drapkin MS: Clinical findings and diagnosis in human granulocytic anaplasmosis: a case series from Massachusetts. Mayo Clin Proc 2012, 87:233-239.
6. Li H, Zhou Y, Wang W, Guo D, Huang S, Jie S: The clinical characteristics and outcomes of patients with human granulocytic anaplasmosis in China. Int J Infect Dis 2011, 15:e859-e866.

7. Eberts MD, Beall MJ, Stillman BA, Chandrashekar R, Breitschwerdt EB: Typical and atypical manifestations of Anaplasma phagocytophilum infection in dogs. J Am Anim Hosp Assoc 2011, 47:e86-e94.

8. Bowman D, Little SE, Lorentzen L, Shields J, Sullivan MP, Carlin EP: Prevalence and geographic distribution of Dirofilaria immitis, Borrelia burgdorferi, Ehrlichia canis, and Anaplasma phagocytophilum in dogs in the United States: results of a national clinic-based serologic survey. Vet Parasitol 2009, 160:138-148.

9. Foley J, Nieto NC, Madigan J, Sykes J: Possible differential host tropism in Anaplasma phagocytophilum strains in the Western United States. Ann N Y Acad Sci 2008, 1149:94-97.

10. Foley JE, Nieto NC, Massung R, Barbet A, Madigan J, Brown RN: Distinct ecologically relevant strains of Anaplasma phagocytophilum. Emerg Infect Dis 2009, 15:842-843.

11. Massung RF, Mather TN, Priestley RA, Levin ML: Transmission efficiency of the AP-variant 1 strain of Anaplasma phagocytophila. Ann N Y Acad Sci 2003, 990:75-79.

12. Massung RF, Priestley RA, Miller NJ, Mather TN, Levin ML: Inability of a variant strain of Anaplasma phagocytophilum to infect mice. J Infect Dis 2003, 188:1757-1763.

13. Massung RF, Courtney JW, Hiratzka SL, Pitzer VE, Smith G, Dryden RL: Anaplasma phagocytophilum in white-tailed deer. Emerg Infect Dis 2005, 11:1604-1606.

14. Stuen S: Anaplasma phagocytophilum - the most widespread tick-borne infection in animals in Europe. Vet Res Commun 2007, 31(Suppl 1):79-84.

15. Dunning Hotopp JC, Lin M, Madupu R, Crabtree J, Angiuoli SV, Eisen J, et al: Comparative genomics of emerging human ehrlichiosis agents. PLOS Genet 2006, 2:e21.

16. Rikihisa Y, Lin M, Niu H: Type IV secretion in the obligatory intracellular bacterium Anaplasma phagocytophilum. Cell Microbiol 2010, 12:1213-1221.

17. Waksman G, Fronzes R: Molecular architecture of bacterial type IV secretion systems. Trends Biochem Sci 2010, 35:691-698.

18. Niu H, Rikihisa Y, Yamaguchi M, Ohashi N: Differential expression of VirB9 and VirB6 during the life cycle of Anaplasma phagocytophilum in human leucocytes is associated with differential binding and avoidance of lysosome pathway. Cell Microbiol 2006, 8:523-534.

19. Ge Y, Rikihisa Y: Identification of novel surface proteins of Anaplasma phagocytophilum by affinity purification and proteomics. J Bacteriol 2007, 189:7819-7828.

20. Alvarez-Martinez CE, Christie PJ: Biological diversity of prokaryotic type IV secretion systems. Microbiol Mol Biol Rev 2009, 73:775-808.

21. Lopez JE, Palmer GH, Brayton KA, Dark MJ, Leach SE, Brown WC: Immunogenicity of Anaplasma marginale type IV secretion system proteins in a protective outer membrane vaccine. Infect Immun 2007, 75:2333-2342.

22. Morse K, Norimine J, Palmer GH, Sutten EL, Baszler TV, Brown WC: Association and evidence for linked recognition of type IV secretion system proteins VirB9-1, VirB9-2, and VirB10 in Anaplasma marginale. Infect Immun 2012, 80:215-227.

23. Morse K, Norimine J, Hope JC, Brown WC: Breadth of the CD4(+) T cell response to Anaplasma marginale VirB9-1, VirB9-2 and VirB10 and MHC class II DR and DQ restriction elements. Immunogenetics 2012, 64:507-523.

24. Araujo FR, Costa CM, Ramos CA, Farias TA, Souza II, Melo ES, et al: IgG and IgG2 antibodies from cattle naturally infected with Anaplasma marginale recognize the recombinant vaccine candidate antigens VirB9, VirB10, and elongation factor-Tu. Mem Inst Oswaldo Cruz 2008, 103:186-190.

25. Sutten EL, Norimine J, Beare PA, Heinzen RA, Lopez JE, Morse K, et al: Anaplasma marginale type IV secretion system proteins VirB2, VirB7, VirB11, and VirD4 are immunogenic components of a protective bacterial membrane vaccine. Infect Immun 2010, 78:1314-1325.

26. Dark MJ, Al-Khedery B, Barbet AF: Multistrain genome analysis identifies candidate vaccine antigens of Anaplasma marginale. Vaccine 2011, 29:4923-4932

27. Park J, Kim KJ, Choi KS, Grab DJ, Dumler JS: Anaplasma phagocytophilum AnkA binds to granulocyte DNA and nuclear proteins. Cell Microbiol 2004, 6:743-751. 
28. Garcia-Garcia JC, Rennoll-Bankert KE, Pelly S, Milstone AM, Dumler JS: Silencing of host cell CYBB gene expression by the nuclear effector AnkA of the intracellular pathogen Anaplasma phagocytophilum. Infect Immun 2009, 77:2385-2391.

29. Niu H, Kozjak-Pavlovic V, Rudel T, Rikihisa Y: Anaplasma phagocytophilum Ats-1 is imported into host cell mitochondria and interferes with apoptosis induction. PLoS Pathog 2010, 6:e1000774.

30. Gillespie JJ, Brayton KA, Williams KP, Diaz MA, Brown WC, Azad AF, Sobral BW: Phylogenomics reveals a diverse Rickettsiales type IV secretion system. Infect Immun 2010, 78:1809-1823.

31. Ohashi N, Zhi N, Lin Q, Rikihisa Y: Characterization and transcriptional analysis of gene clusters for a type IV secretion machinery in human granulocytic and monocytic ehrlichiosis agents. Infect Immun 2002, 70:2128-2138.

32. Berger BR, Christie PJ: Genetic complementation analysis of the Agrobacterium tumefaciens virB operon: virB2 through virB11 are essential virulence genes. J Bacteriol 1994, 176:3646-3660.

33. Yuan Q, Carle A, Gao C, Sivanesan D, Aly KA, Hoppner C, et al: Identification of the VirB4-VirB8-VirB5-VirB2 pilus assembly sequence of type IV secretion systems. J Biol Chem 2005, 280:26349-26359.

34. Judd PK, Kumar RB, Das A: Spatial location and requirements for the assembly of the Agrobacterium tumefaciens type IV secretion apparatus. Proc Natl Acad Sci USA 2005, 102:11498-11503.

35. Kumar RB, Xie YH, Das A: Subcellular localization of the Agrobacterium tumefaciens T-DNA transport pore proteins: VirB8 is essential for the assembly of the transport pore. Mol Microbiol 2000, 36:608-617.

36. Cascales E, Christie PJ: Agrobacterium VirB10, an ATP energy sensor required for type IV secretion. Proc Natl Acad Sci USA 2004, 101:17228-17233.

37. Rances E, Voronin D, Tran-Van V, Mavingui P: Genetic and functional characterization of the type IV secretion system in Wolbachia. J Bacteriol 2008, 190:5020-5030.

38. Anderson LB, Hertzel AV, Das A: Agrobacterium tumefaciens VirB7 and VirB9 form a disulfide-linked protein complex. Proc Natl Acad Sci USA 1996, 93:8889-8894.

39. Spudich GM, Fernandez D, Zhou XR, Christie PJ: Intermolecular disulfide bonds stabilize VirB7 homodimers and VirB7/VirB9 heterodimers during biogenesis of the Agrobacterium tumefaciens T-complex transport apparatus. Proc Natl Acad Sci USA 1996, 93:7512-7517.

40. Bayliss R, Harris R, Coutte L, Monier A, Fronzes R, Christie PJ, et al: NMR structure of a complex between the VirB9/VirB7 interaction domains of the pKM101 type IV secretion system. Proc Natl Acad Sci USA 2007, 104:1673-1678.

41. Ge Y, Rikihisa Y: Surface-exposed proteins of Ehrlichia chaffeensis. Infect Immun 2007, 75:3833-3841.

42. Meeus PF, Brayton KA, Palmer GH, Barbet AF: Conservation of a gene conversion mechanism in two distantly related paralogues of Anaplasma marginale. Mol Microbiol 2003, 47:633-643.

43. Dumler JS, Barbet AF, Bekker CP, Dasch GA, Palmer GH, Ray SC, et al: Reorganization of genera in the families Rickettsiaceae and Anaplasmataceae in the order Rickettsiales: unification of some species of Ehrlichia with Anaplasma, Cowdria with Ehrlichia and Ehrlichia with Neorickettsia, descriptions of six new species combinations and designation of Ehrlichia equi and 'HGE agent' as subjective synonyms of Ehrlichia phagocytophila. Int J Syst Evol Microbio/ 2001 51:2145-2165.

44. Gribble DH: Equine ehrlichiosis. J Am Vet Med Assoc 1969, 155:462-469.

45. Johnson RC, Kodner C, Jarnefeld J, Eck DK, Xu Y: Agents of human anaplasmosis and Lyme disease at Camp Ripley, Minnesota. Vector Borne Zoonotic Dis 2011, 11:1529-1534.

46. Munderloh UG, Jauron SD, Fingerle V, Leitritz L, Hayes SF, Hautman JM, et al: Invasion and intracellular development of the human granulocytic ehrlichiosis agent in tick cell culture. J Clin Microbio/ 1999, 37:2518-2524.

47. Massung RF, Levin ML, Munderloh UG, Silverman DJ, Lynch MJ, Gaywee JK Kurtti TJ: Isolation and propagation of the Ap-Variant 1 strain of Anaplasma phagocytophilum in a tick cell line. J Clin Microbiol 2007, 45:2138-2143.

48. Granquist EG, Bardsen K, Bergstrom K, Stuen S: Variant and individual dependent nature of persistent Anaplasma phagocytophilum infection. Acta Vet Scand 2010, 52:25
49. Felsheim RF, Herron MJ, Nelson CM, Burkhardt NY, Barbet AF, Kurtti TJ, Munderloh UG: Transformation of Anaplasma phagocytophilum. BMC Biotechnol 2006, 6:42

50. Katoh K, Toh H: Recent developments in the MAFFT multiple sequence alignment program. Brief Bioinform 2008, 9:286-298.

51. Goodstadt L, Ponting CP: CHROMA: consensus-based colouring of multiple alignments for publication. Bioinformatics 2001, 17:845-846.

52. Jones DT, Taylor WR, Thornton JM: The rapid generation of mutation data matrices from protein sequences. Comput Appl Biosci 1992, 8:275-282.

53. Hopp TP, Woods KR: A computer program for predicting protein antigenic determinants. Mol Immunol 1983, 20:483-489.

54. Hopp TP: Use of hydrophilicity plotting procedures to identify protein antigenic segments and other interaction sites. Methods Enzymol 1989, 178:571-585.

55. Goecks J, Nekrutenko A, Taylor J: Galaxy: a comprehensive approach for supporting accessible, reproducible, and transparent computational research in the life sciences. Genome Biol 2010, 11:R86.

doi:10.1186/1471-2164-13-678

Cite this article as: Al-Khedery et al: Structure of the type IV secretion system in different strains of Anaplasma phagocytophilum. BMC Genomics 2012 13:678.

\section{Submit your next manuscript to BioMed Central and take full advantage of:}

- Convenient online submission

- Thorough peer review

- No space constraints or color figure charges

- Immediate publication on acceptance

- Inclusion in PubMed, CAS, Scopus and Google Scholar

- Research which is freely available for redistribution 